\title{
Effect of Double Suprarenalectomy on the Blood Coagulation Time in Cats.
}

\author{
By \\ FUMIO OHMI. \\ (近 江 交男) \\ (From the Physiological Laboratory of Prof. Y. Satake, \\ Tohoku Imperial University, Sendai.)
}

The heading of this paper is a mere repetition of that of one published by Barlow and Ellis ${ }^{1)}$ about ten years ago, and the contents also.

The authors noted that the blood coagulation time in cats became consistently shorter after double suprarenalectomy and progressively shorter as the death point was approached. The present writer has had an opportunity to test this.

Similar findings were given with rabbits; $\mathrm{Okada}^{2}$ extirpated the suprarenals by the lumbar way in rabbits which were capable of surviving only two to twelve days. The blood coagulation time, estimated by B rodi e's coagulometer, was 4 to 4.5 minutes before the removal and 2 to 2.5 minutes after. Ichikawa $a^{3}$ made use of the coagulometer of Biffi-Brooks-Sato and attempted to remove the glands in rabbits in one or two sittings. In six rabbits, the glands of which were removed in one sitting, the coagulation time tested 24 hours to 21 days or so was noted as shorter than before, but when tested 20 days (Rabbit No. 4) or 58 days (No. 6) not only no shortening was noted, but rather a prolongation. Those operated on in two sittings yielded rather inconstant results; it is difficult to take the data from two out of six (Nos. $2 \& 4$ ) as harmonizing with the above related features.

The coagulation time in the doubly suprarenalectomized rabbits will be discussed on a later occasion by Doctor Sh. K a n o w ok a who has had an opportunity of carrying out this kind of experiment.

Cats, male as well as female, were used, and never fastened or anaesthetized for estimating the coagulation time. Blood samples were collected from the posterior branch of the marginal vein of the

1) Barlow and Ellis, Am. Jour. of Physiol., 1924, 70, 58.

2) $0 \mathrm{k}$ a da, Nippon Naibumpitsu-Gakkai Zasshi, 1927(-28), 3, 110 (Proc.).

3) Ichik a wa, Ibid., 1929(-30), 5, 1515 (German referat p. 87.). 
ear previously denervated, and the determination of the coagulation time was made by means of the apparatus of Brodie, Russel and Boggs. It may be further added that in carrying out the determinations the first few or some samples clotted rather more readily than the subsequent ones. Ten or twenty samples were taken with a regular interval of 5 minutes, and their values were averaged. Suprarenal glands were removed under ether through the lumbar route in two sittings; the right gland was removed first and after 30 days or later the left one.

Of 8 cats (Cats Nos. $1 \& 9-15$ ) the coagulation time of blood was determined before the glands had been removed, and of the remainder, 7 in number, after the right gland only had been extirpated, ample time being allowed to elapse between the removal and the coagulation experiment. The values for the former set were 1.6 to 2.6 minutes, mean 1.8 minutes and those for the latter 1.8 to 2.4 minutes, mean 2.1 minutes, therefore the unilateral suprarenalectomy has no influence. upon the coagulation time at all. Taking the values altogether, the blood coagulation time of normal cats, determined by the Brodie, is 1.6 to 2.6 minutes, mean 1.9 or smoothly 2 minutes.

After the last decapsulation under ether, the animals were not especially treated, and the coagulation tests were made at an interval of about 24 hours ; no determinations were made on the day of decapsulation, because of the possibility of some influence of anaesthetization and operation upon the coagulation.

As is to be seen from the Table the cats lived two to six days after the total decapsulatien; most of them three or four days.

On the next day after the last removal the coagulation time was found remarkably shortened, the average values of all the cats being noted as 0.6-1.4 minutes, mean 0.9 minutes, that is about half of the initial. Only the shortening was insignificant in 4 cases (Nos. 4, 9, $13 \& 15)$; the figures before 1.7-1.8 minutes and thereafter 1-1.4 minutes. One day later the determinations were carried out with 14 cats, the coagulation time became only a little shorter on an average, that is in half of the cases no further shortening occurred. The estimations gave the figures as $0.5-1.35$ minutes, mean 0.7 minutes. That of Cats Nos. $4 \& 9$ remained as before, while that of Cats Nos. $13 \&$ 15 became decidedly shorter. Three days after the decapsulation we could make the determinations with 7 cats only, the same number of cats were meanwhile lost. Cat 9 showed a still somewhat long time of coagulation as 1.1 minutes on an average, while the rest showed 0.5 minutes as the coagulation time. Four days after the decapsulation 4 cats were living, they gave the average coagulation time of 0.5 minutes; and one cat (No. 8) was capable of surviving one day more. 


\author{
Experiment 8. \\ Cat ô.
}

7. VII. 1930. 3.48 kilos. R suprarenalectomy and auricular nerve severed.

16. VII. 3.40 kilos. Anal temperature $38.1^{\circ} \mathrm{C}$.; Room temperature $12.5^{\circ} \mathrm{C}$. Coagulation time taken. (1)

18. XI. 3.35 kilos. L suprarenalectomy.

19. XI. 3.17 kilos. Anal temperature $37.5^{\circ} \mathrm{C}$; Room temperature $16^{\circ} \mathrm{C}$. Coagulation time taken. (2)

20. XI. 3.15 kilos. Anal temperature $37.1^{\circ} \mathrm{C}$; Room temperature $17^{\circ} \mathrm{C}$. Coagulation time taken. (3)

21. XI. 3.13 kilos. Anal temperature $36.5^{\circ} \mathrm{C}$; Room temperature $19^{\circ} \mathrm{C}$. Coagulation time taken. (4)

22. XI. 3.05 kilos. Anal temperature $34.5^{\circ} \mathrm{C}$; Room temperature $17^{\circ} \mathrm{C}$. Coagulation time taken. (5)

23. XI. 2.97 kilos. Anal temperature $32.0^{\circ} \mathrm{C}$; Room temperature $16^{\circ} \mathrm{C}$. Coagulation time taken. (6)

\begin{tabular}{|c|c|c|c|c|c|c|c|c|c|c|c|}
\hline \multirow{2}{*}{\multicolumn{2}{|c|}{$\begin{array}{c}\begin{array}{c}\text { Before suprare- } \\
\text { nalectomy }\end{array} \\
\text { (1) } 16 . \mathrm{VII} .\end{array}$}} & \multicolumn{10}{|c|}{ After suprarenalectomy } \\
\hline & & \multicolumn{2}{|c|}{ (2) $19 . \mathrm{XI}$. } & \multicolumn{2}{|c|}{ (3) $20 . \mathrm{XI}$. } & \multicolumn{2}{|c|}{ (4) 21. XI. } & \multicolumn{2}{|c|}{ (5) $22 . \mathrm{XI}$. } & \multicolumn{2}{|c|}{ (6) 23. XI. } \\
\hline 㤩 & 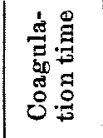 & $\underset{\Xi}{\stackrel{g}{E}}$ & 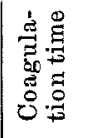 & $\underset{\xi}{\stackrel{g}{\xi}}$ & 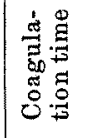 & 兽 & 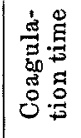 & $\stackrel{\mathbb{E}}{\mathrm{E}}$ & 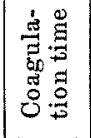 & $\underset{:}{\stackrel{\Xi}{E}}$ & 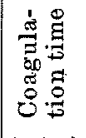 \\
\hline (h.:m.) & $(\min )$. & $(\mathrm{h} .: \mathrm{m})$. & (min.) & (h.: m. & $(\min )$. & $(\mathrm{h} . \mathrm{m}$.) & $(\min )$. & $(\mathrm{h} .: \mathrm{m})$. & $(\min )$. & $(\mathrm{h} . \mathrm{m})$. & $(\min )$. \\
\hline a.m. & & p.m & & p.m & & p.m & & p.m. & & p.m. & \\
\hline $9: 35$ & 3.5 & $1: 15$ & 1.5 & $2: 20$ & 1.5 & 3:0 & 1.0 & $2: 30$ & 0.5 & $1: 50$ & 0.5 \\
\hline$: 40$ & 3.0 & $: 2$ & 1.5 & $: 25$ & 1.0 & $: 5$ & $1.0-$ & $: 35$ & 0.5 & $: 55$ & $0.5-$ \\
\hline$: 45$ & 2.0 & :25 & 1.0 & $: 30$ & $1.0^{-}$ & :10 & 0.5 & $: 4$ & 0.5 & 2: 0 & 0.5 \\
\hline$: 50$ & 1.5 & $: 30$ & 0.5 & $: 35$ & 0.5 & $: 16$ & 0.5 & $: 45$ & 0.5 & $: 5$ & 0.5 \\
\hline$: 55$ & 2.5 & :35 & 0.5 & $: 40$ & 0. & $: 20$ & 0. & $: 50$ & 0.5 & :10 & 0.5 \\
\hline 10: 0 & 2.5 & $: 40$ & 0.5 & $: 45$ & 0. & :25 & 0.5 & $: 55$ & $0.5^{-}$ & $: 15$ & 0.5 \\
\hline$: 5$ & 2.0 & $: 45$ & 0.5 & $: 50$ & 0. & $: 30$ & 0.5 & 3: 0 & 0.5 & :20 & 0.6 \\
\hline :10 & 2.5 & $: 50$ & 0.5 & $: 55$ & 0.5 & $: 35$ & 0.5 & $: 5$ & $0.5^{-}$ & :25 & 0.5 \\
\hline$: 15$ & 1.5 & $: 55$ & 0.5 & 3: 0 & 0.5 & $: 40$ & 0.5 & & 0.5 & $: 30$ & 0.5 \\
\hline$: 20$ & 2.0 & 2: 0 & 0.5 & $: 5$ & 0.5 & $: 45$ & $\begin{array}{l}0.5 \\
\end{array}$ & $: 15$ & $0.5-$ & :35 & 0.5 \\
\hline Mean & $\begin{array}{c}(1.5-3.5) \\
2.4\end{array}$ & & $\begin{array}{c}.5-1.5) \\
0.75\end{array}$ & & $\begin{array}{r}0.5-1.5 \\
0.7\end{array}$ & & $\begin{array}{c}0.5-1.0 \\
0.6\end{array}$ & & $\begin{array}{c}-0.5 \\
0.5\end{array}$ & & $\begin{array}{c}-0.5) \\
0.5\end{array}$ \\
\hline
\end{tabular}

It must be said by way of precaution that the test whether the blood is clotted or not was made every thirty seconds; when the clot was very hard it was noted less than 0.5 minutes, with an expression $0.5^{-}$ in the Table and the example.

Thus it was ascertained that the blood coagulation time of cats becomes decidedly shorter on the day following the double suprarenalectomy, and afterwards the shortening progresses further, though slowly. 
$\mathrm{T}_{\mathrm{A}}$

Coagulation time in cats before and

\begin{tabular}{|c|c|c|c|c|c|c|c|c|c|c|c|c|c|}
\hline \multirow{3}{*}{ 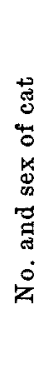 } & \multicolumn{5}{|c|}{$\begin{array}{l}\text { Before first or second } \\
\text { suprarenalectomy }\end{array}$} & \multicolumn{4}{|c|}{ Suprarenalectomy } & & & & \\
\hline & \multirow{2}{*}{ 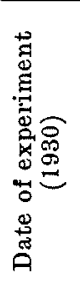 } & \multirow{2}{*}{ 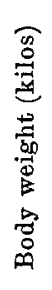 } & \multirow{2}{*}{ 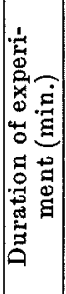 } & \multirow[b]{2}{*}{ 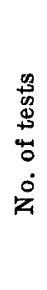 } & \multirow[b]{2}{*}{ 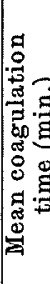 } & \multicolumn{2}{|c|}{$\begin{array}{l}\text { Right } \\
\text { gland }\end{array}$} & \multicolumn{2}{|c|}{$\begin{array}{l}\text { Left } \\
\text { gland }\end{array}$} & \multicolumn{2}{|c|}{$\begin{array}{c}\text { Estimation of } \\
\text { coagulation time }\end{array}$} & \multicolumn{2}{|c|}{$\begin{array}{l}\text { Body tem- } \\
\text { perature }\end{array}$} \\
\hline & & & & & & 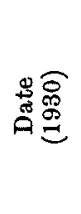 & 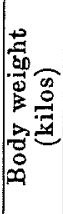 & 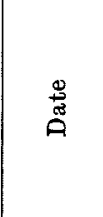 & 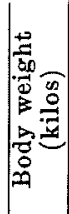 & 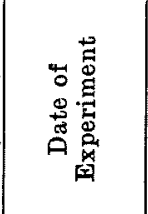 & 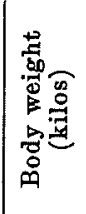 & 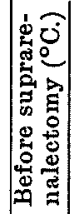 & 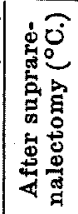 \\
\hline 1 & 17. & & & & & & & & & & & & \\
\hline$\hat{o}$ & & 3.10 & 90 & 20 & 2.6 & VI. & 2.86 & & & $x, 30$ & 2.56 & 38.9 & \\
\hline 2 & 14. & 2.60 & $"$ & $"$ & 2.2 & 7 & 2.52 & & & 15.7 & & 38.7 & 37.2 \\
\hline $\begin{array}{l}0 \\
3 \\
3\end{array}$ & & & & & & & & & & ' 30 & & & \\
\hline$\hat{o}$ & VII. & 2.85 & $"$ & $"$ & $2.0^{\prime}$ & VI. & 3.12 & & & $\mathrm{X} .30$ & & 8.9 & 0 \\
\hline $\begin{array}{l}4 \\
9\end{array}$ & $\begin{array}{l}16 . \\
\text { VII. }\end{array}$ & 2.48 & 45 & 10 & 1.8 & $\begin{array}{l}21 . \\
\text { VI. }\end{array}$ & 2.68 & & 2.45 & $\left|\begin{array}{l}\text { 8. X. } \\
\text { 9. X.' } 30\end{array}\right|$ & $\begin{array}{r}2.43- \\
2.30\end{array}$ & 38.9 & \\
\hline 5 & 17. & 2.70 & 90 & 20 & 1.8 & 21. & 2.84 & & 2.77 & 26. XI- & $2.66-$ & 39.0 & \\
\hline$\frac{7}{6}$ & & & & & & & & & & & $2.56-$ & & \\
\hline s & VII. & & $"$ & $"$ & & V. & 2.28 & & & $\mathrm{x} . ' 30$ & & 39.1 & \\
\hline 7 & 25 & 2.53 & 45 & 10 & 2.1 & 9. & .53 & & & 6.2 & $2.44-$ & 38.6 & \\
\hline $\begin{array}{l}\hat{0} \\
8\end{array}$ & IV & & & & & IX. & & & & .'30 & 2.10 & & \\
\hline$\hat{o}$ & VII & 3.40 & $"$ & $"$ & 2.4 & vir. & 3.48 & & 3.35 & & & 38.1 & \\
\hline 9 & 18 & 05 & & & $7 !$ & 18. & & & & & & & \\
\hline 3 & $\mathrm{X}$ & 2.00 & $"$ & $"$ & 1.7 & XI. & .00 & & 27 & II.'30 & 1.95 & 38.9 & 33. \\
\hline 10 & $\mathrm{x}$ & 3.11 & " & & 1.6 & $\mathbf{2 .}$ & 3.11 & & 2.83 & 3. II. - & $2.68-$ & 38.0 & 34.0 \\
\hline 11 & & & & & & & & & & & & & \\
\hline & $\mathrm{XI}$ & & $"$ & $"$ & 1 & XII. & & & 2.78 & & & 38.0 & 34. \\
\hline 12 & 1. & & & & & & & & & & & & $\mathbf{8 5 . 4}$ \\
\hline & & & $"$ & " & & & & & & & 2.10 & & 00.4 \\
\hline 13 & 16 & 2.04 & $"$ & & 1.8 & 16. & 2.04 & & 2.00 & 10. II. - , & $1.98-$ & 38.4 & 38.4- \\
\hline 14 & & & & & & & & & & 1 & $2.38-$ & & \\
\hline & $\mathrm{XI}$ & & $"$ & , & 1 & XII. & & & & & 2.33 & 3.5 & 34.5 \\
\hline 15 & 2 & 2.65 & & $"$ & 1.7 & 23. & 2.65 & 16. & 2.45 & 17. II. - & $2.43-$ & 38.0 & $37.0-$ \\
\hline & $X$ & & & & & XII. & & & & & 2.38 & & \\
\hline
\end{tabular}


BLE.

after double suprarenalectomy.

After double suprarenalectomy

\begin{tabular}{|c|c|c|c|c|c|c|c|c|c|c|c|c|c|c|c|}
\hline \multirow[b]{2}{*}{ 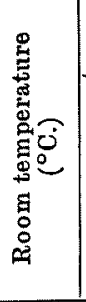 } & \multicolumn{3}{|c|}{$\begin{array}{l}\text { 1st day of experi. } \\
\text { ment, next day of } \\
\text { suprarenalectomy }\end{array}$} & \multicolumn{3}{|c|}{ 2nd day } & \multicolumn{3}{|c|}{ 3rd day } & \multicolumn{3}{|c|}{ 4th day } & \multicolumn{3}{|c|}{ 5th day } \\
\hline & 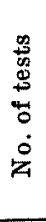 & 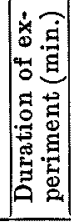 & 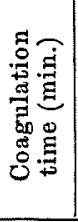 & 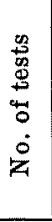 & 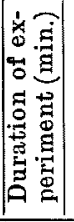 & 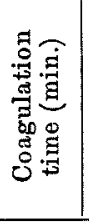 & 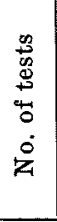 & 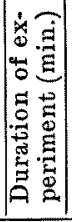 & 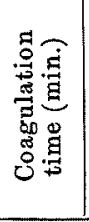 & 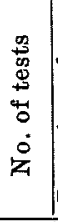 & 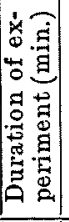 & 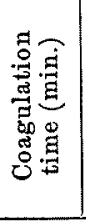 & 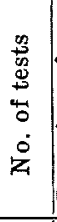 & 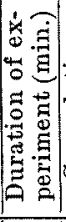 & 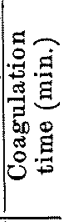 \\
\hline $\begin{array}{l}13- \\
12.5\end{array}$ & 10 & 45 & $0.6^{-}$ & 10 & 45 & 0.55 & 10 & 45 & $0.5-$ & & & & & & \\
\hline $17-16$ & $"$ & $"$ & 0.6 & $"$ & $"$ & 0.65 & $"$ & $"$ & $0.55-$ & & & & & & \\
\hline $16-13$ & $"$ & $"$ & $0.8^{+}$ & $"$ & $"$ & 0.55 & $"$ & $"$ & $0.5-$ & & & & & & \\
\hline $17-16$ & $"$ & $"$ & 1.25 & $"$ & $"$ & 1.35 & & & & & & & & & \\
\hline 21-17 & $"$ & $"$ & $0.8-$ & $"$ & $"$ & $0.6^{-}$ & 10 & 45 & $0.6^{-}$ & 10 & 45 & $0.55-$ & & & \\
\hline 17 & $"$ & $"$ & 0.8 & $"$ & $"$ & $0.7-$ & & & & & & & & & \\
\hline 20-16 & $"$ & $"$ & 0.9 & $"$ & $"$ & $0.55-$ & & & & & & & & & \\
\hline $19-16$ & $"$ & $"$ & 0.75 & $"$ & $"$ & $0.7-$ & 10 & 45 & 0.65 & 10 & 45 & $0.5^{-}$ & 10 & 45 & $0.5^{-}$ \\
\hline $20-16$ & $"$ & $"$ & 1.4 & $"$ & $"$ & 1.4 & $"$ & $"$ & 1.1 & $"$ & $"$ & 0.55 & & & \\
\hline 16 & $"$ & $"$ & 0.75 & $"$ & $"$ & $0.6^{-}$ & & & & & & & & & \\
\hline 20-19 & $"$ & $"$ & 0.8 & $"$ & $"$ & $0.5^{-}$ & & & & & & & & & \\
\hline 21 & $"$ & $"$ & 0.65 & & & & & & & & & & & & \\
\hline $20-18$ & $"$ & $"$ & 1.3 & 10 & 45 & $0.55_{-}$ & 6 & 25 & $0.4^{-}$ & 5 & 20 & $0.5^{-}$ & & & \\
\hline $22-20$ & $"$ & $"$ & $0.7-$ & 7 & 30 & $0.7-$ & & & & & & & & & \\
\hline 19 & $"$ & $"$ & 1.0 & 9 & 40 & $0.55-$ & & & & & & & & & \\
\hline
\end{tabular}

